\title{
Fostering Apprentice Beauticians' Visual Expertise Through Annotations: A Design Experiment Using the Platform Realto
}

\author{
Alessia Eletta Coppi ${ }^{1}$, Alberto Cattaneo ${ }^{1}$ \\ ${ }^{1}$ Swiss Federal Institute for Vocational Education and Training, Switzerland \\ Correspondence: Alessia Eletta Coppi, PhD student, Swiss Federal Institute for Vocational Education and Training, \\ Switzerland.
}

Received: May 31, 2021

Accepted: June 27, 2021

Online Published: July 12, 2021

doi:10.11114/jets.v9i7.5291

URL: https://doi.org/10.11114/jets.v9i7.5291

\begin{abstract}
Professional beauticians regularly perform skin analyses and should be skilled at observing small skin anomalies and skin damages. However, little is done directly for improving their observation skills at school, during their training. In order to foster apprentices' observation skills of skin anomalies, a new training scheme exploiting annotations and attentionguiding methods through the use of the platform Realto was developed and tested.

A second year class of apprentice beauticians $(\mathrm{N}=9)$ was given a pre-test on the visual expertise of skin anomalies. Then, for a semester they attended multiple training sessions where firstly teachers explained skin anomaly images with the help of annotations (attention-guiding) and secondly, students were asked to observe other images of the same anomalies, annotate them, and then provide a textual description of the identified anomaly.

At the end of the semester, the trained class completed a post-test and a questionnaire, and group interview have been collected. Another group of apprentices $(\mathrm{N}=19)$ of the third year who already completed the skin anomaly course was used as baseline. Results showed that the group who participated in the treatment mentioned almost double the amount of details noticed by the baseline group in the post-test; being trained using annotations and the Realto platform proved effective in developing observation skills, compared to normal lessons. Furthermore, apprentices confirmed, both through the questionnaire and the interviews, that they considered annotations useful for improving their observation skills and that using Realto and its annotation facilities was a good way to achieve this result.
\end{abstract}

Keywords: vocational education and training; annotation; visual expertise skill; cueing; technology-enhanced learning environment

\section{Introduction}

Visual expertise skills are essential in many occupations, such as engineering (e.g., Katsioloudis \& Stefaniak, 2018), medicine (e.g., Naghshineh et al., 2008), architecture (e.g., Styhre \& Gluch, 2009) and sports (e.g., Kredel et al., 2017). However, most studies tend to focus on white-collar professions, neglecting the world of vocational education.

This gap in the research is puzzling because vocations strongly rely on visual skills to perform profession-specific tasks. For instance, to create proper clothing, fashion designers must visually explore images of garments to identify details and defects while keeping specific characteristics of the customer's body in mind (Caruso et al., 2019). Similarly, beauticians must observe the skin of their clients to identify anomalies, provide treatment or offer a cosmetic procedure. This must be done to ensure that a treatment (e.g., pedicure) does not result in injuries and to identify anomalies that require direct medical inspection (e.g., moles with irregular edges or dark black or irritated moles) before any cosmetic procedure (State Secretariat for Education, Research and Innovation, 2007). This is a complex task because various skin anomalies can look similar to each other, which can lead to a diagnostic error and the wrong treatment choice, ultimately leading to potential skin damage.

The current study investigates how to promote observation skills in beauticians' training, with the main goal of fostering visual expertise in observing skin anomalies in apprentice beauticians and increasing their accuracy rate by using a variety of methods (e.g., repeated exposure to annotation of anomaly images).

In the following section, literature on visual expertise-particularly in the dermatological area-will be explored. This line of literature is explored because of the similar knowledge that beauticians and dermatologists share when it comes to skin anomalies. Following this, the literature on methods to foster visual expertise skills will be explored and discussed. 


\subsection{Visual Expertise Skills in Dermatological Areas}

Visual expertise requires individuals to enact different types of processes. The perceptual processes underlying visual expertise are fundamental because professionals cannot only rely on procedural and declarative knowledge (Kellman \& Krasne, 2018) to perform their tasks. These processes can be divided into the following four stages: 1) identification of things that are worthy of further analysis, 2) recognition of relevant sections of images, 3) identification of target sections (e.g., lesions) and 4) diagnosis (Waite et al., 2019). For example, perceptual learning allows professional radiologists to perform visual searches in X-rays and process information to interpret lesions (Ravesloot et al., 2012), as well as recognise patterns of symmetry, colours and shapes (Shapiro et al., 2006). It also helps artists shift between the local and global processing (Chamberlain \& Wagemans, 2015) of images.

Much like beauticians, dermatologists perform tasks such as skin analyses and visual recognition of skin conditions or abnormalities using two approaches. The first system is perceptual and involves the automatic gathering of information, which includes fast and intuitive thinking, pattern recognition and the use of heuristics. The second system is slow, logical, analytical and, most of all, deliberate and conscious. With the first system, dermatologists can 1) recognise anomalies, even if they only see a part of them (e.g., unilateral and clustered symptoms), 2) group objects that are close to each other (e.g., simultaneous presence of telangiectasia, papules, macules and hypopigmented area being a manifestation of Goltz syndrome), 3) identify anomalies by a specific colour (e.g., erythema can vary from light pink to red) and 4) separate healthy from unhealthy skin (e.g., figure separation). Other perceptual elements involved in the first system that can help in identifying lesions are 1) the pattern or silhouette of the outline of the lesion, 2) location since lesions tend to appear only in specific areas and 3) contextual information (e.g., age and gender) (Lowenstein et al., 2019). The second system uses high-level processing, which is characterised by mnemonics, algorithms, a checklist and a list of diagnoses (Ko et al., 2019).

\subsection{Improving and Supporting Visual Expertise Skills in Novices}

Improving one's visual expertise is not a new topic and has been explored using different approaches. For instance, the signalling approach is based on the principle postulated by Mayer (2002), which indicates that any kind of signals, such as arrows, colours or text, applied to presented content will help learners diminish their cognitive load while also identifying the relevant targets among the irrelevant ones much faster than without any cues. These signals or annotations can assume different forms, such as colours, text, gestures, flashlights (Schneider et al., 2018), transparent overlay, graphic organisers, shapes and diagrammatic elements (Heiser \& Tversky, 2006), which include free lines (Shin \& Park, 2019; van Gog, 2014), among others. These types of annotations are used by researchers to investigate how to train novices to observe images by directing their gaze and their attention towards the task-relevant and salient parts of content.

For example, in educational contexts, arrows have been shown to be effective when applied to animations (Boucheix \& Lowe, 2010) or colour patches on sports videos for training (D'Innocenzo et al., 2016). Annotations such as circles, colours and highlights have been used effectively on textual content (Stefanut \& Gorgan, 2008; Zywica \& Gomez, 2008). Annotations such as colour shading and circles on X-rays have also proved useful to highlight important elements of fractures (De Koning et al., 2007; Scheiter et al., 2019).

The use of annotations to foster visual expertise in the dermatological area is limited compared with other medical fields (e.g., radiology), but not untraceable. One example is the work of Friedman et al. (1985), who developed a list of criteria (e.g., asymmetry, colour, colour uniformity, border regularity and texture) to diagnose anomalies. This list was used in a study where novices viewed 45 images of anomalies with the help of a sheet (Figure 1) that contained diagnosis criteria, a small drawing and a continuum bar indicating the range of possible presentations of that criteria (e.g., uniform to nonuniform, light to dark, small to large, symmetric to asymmetric, regular to irregular and smooth to rough). These anchors did not have a considerable impact on the diagnostic scores, but the study sample was very small (Zanotto et al., 2011). 


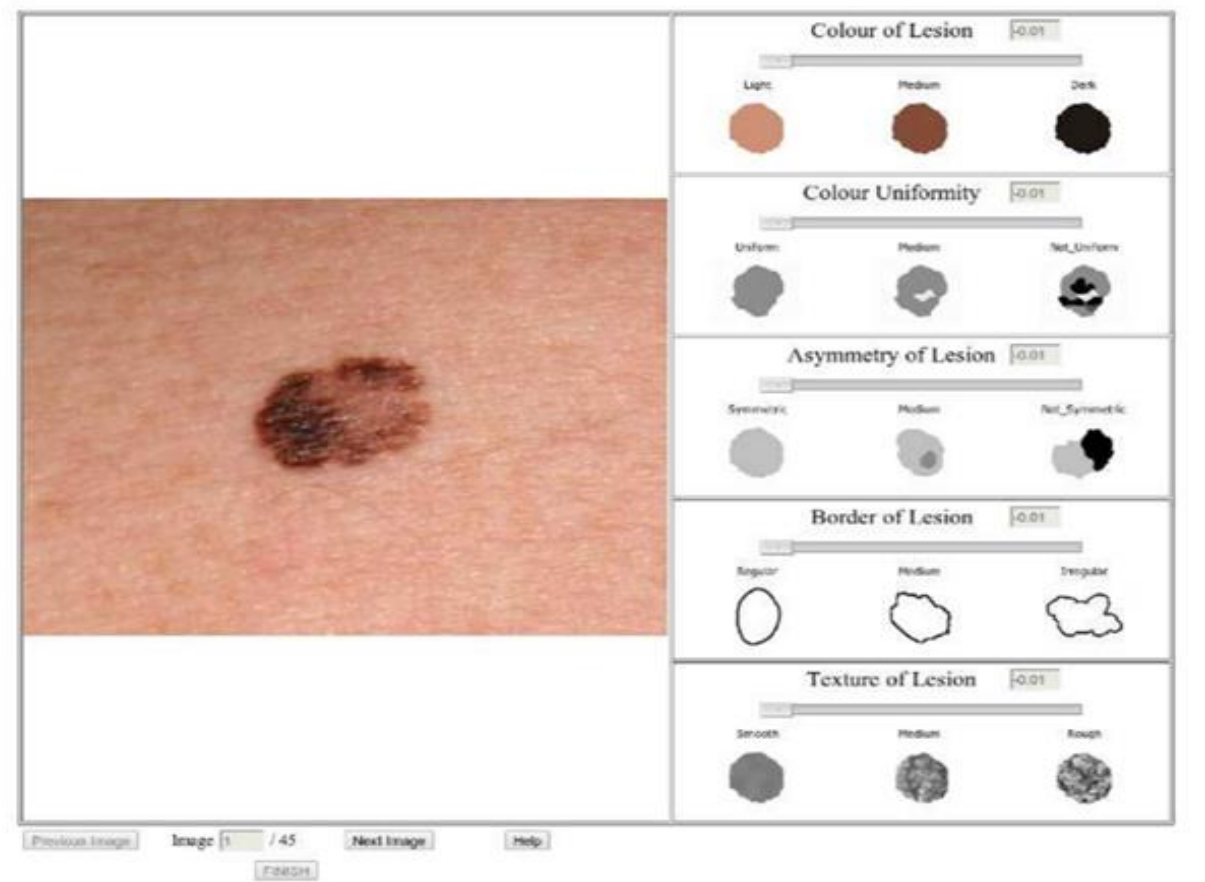

Figure 1. Example of anomaly images and anchors created by Zanotto et al., 2011

Other studies have focused on developing electronic tools to ease the work of professional dermatologists. One example is a tool containing manual segmentation, labelling and image manipulation functions (Ferreira et al., 2012). As a comprehensive tool, LesionMap contains the functions needed to indicate information about anomalies, such as orientation, width, position and opacity (Figure 2; Eapen et al., 2020).

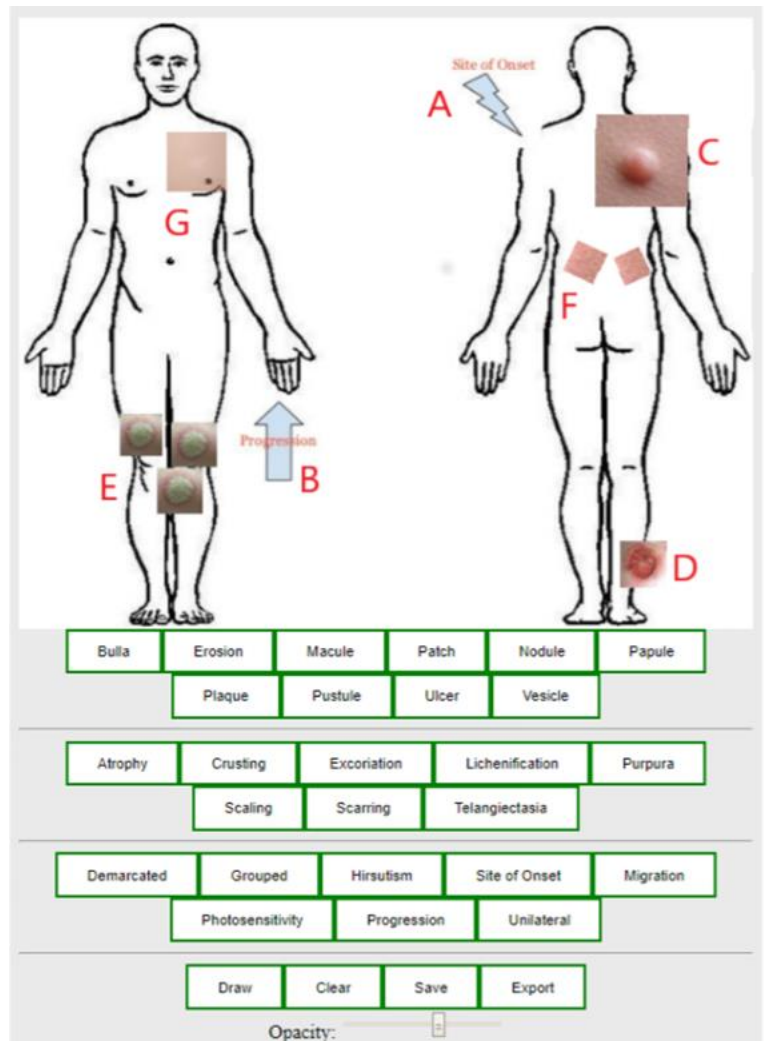

Figure 2. LesionMap developed by Eapen et al., 2012

How to train novices to develop their visual expertise skills, however, is still a question worth investigating. For instance, Dolev et al. (2011) developed eDerm, a curriculum for dermatologists that successfully increased diagnostic rates. eDerm 
contains 17 tutorials and lectures on pigmented and nonpigmented lesions, melanoma, nonmelanon skin cancer, nevi and other benign dermatoses. Other studies have used a memory approach and asked complete novices to match images of anomalies (e.g., basal cell carcinoma, squamous cell carcinoma and seborrheic keratoses) to different sets of images of anomalies (Brown et al., 2009). Similarly, the applied behaviour analysis, precision and training method has been shown to work with a memory approach, based on students who were asked to go through flash cards depicting images of skin anomalies on one side and the names of the anomalies on the other side. This method has proven useful in the recognition of anomalies compared with normal training methods (McGrath et al., 2018). Likewise, perceptual and adaptive learning modules (PALMs) train students by exposing them to digital flashcards containing images of anomalies. Students are shown the images and presented with four possible answers and feedback. The algorithm of PALMs dynamically adjusts the presentation of the images and increases the accuracy of the trainees (Rimoin et al., 2015).

Further, a completely different way to train dermatologists is with art, such as paintings. In fact, with visual thinking strategies, students are trained in general observation through the use of paintings and discussion on topics, such as the meaning of visual expertise and the relationships between observation and communication as well as ambiguity and subjectivity. Evaluations of this method indicate that students' diagnosis and image description skills improved (Zimmermann et al., 2016). Another example of the use of art is seen in the study by Kyriakou and Kyriakou (2021); using Frida Kahlo's paintings, the authors presented examples of dermatological issues that affected the painter to train novices in recognising skin anomalies.

The literature reviewed until this point has indicated a gap in existing studies on enhancing visual expertise in a nonmedical field, especially in vocational professions. This gap can be filled by taking advantage of the unexplored potential and richness of vocational professions relying on visual expertise. Therefore, the present study will focus on fostering apprentice beauticians' observation of skin anomalies by using a training method that combines annotations and written descriptions. This method is based on the literature presented above from the dermatological field.

\subsection{Hypotheses}

Our hypotheses are that students exposed to 1) the use of annotations (teacher-driven annotations, as well as the student's own annotations) to pinpoint skin anomalies and 2) making their own textual descriptions of images of skin anomalies will:

- H1) write more detailed descriptions of skin anomalies at the post-test stage compared with the pre-test stage, after being presented with the images of such anomalies.

- H2) write more detailed descriptions of the images of skin anomalies at the post-test stage than those who completed a traditional course on skin anomalies.

- H3) positively rate the impact and usefulness of annotations and descriptions on visual expertise.

- H4) rate the experience with Realto, specifically its annotation tool, as positive.

\section{Method}

\subsection{Sample}

The sample was composed of 28 students who were all females and enrolled in a vocational certificate programme from a vocational school in Switzerland. The experimental group was composed of 9 apprentices beauticians attending the second year of their course $\left(\mathrm{M}_{\mathrm{age}}=17, \mathrm{SD}=0.75\right)$ and the baseline group was composed of 19 apprentices beauticians enrolled in the third year of their course $\left(\mathrm{M}_{\mathrm{age}}=29.6, \mathrm{SD}=9.83\right)$. The choice of using a second year class as the experimental group was due to the fact that the curriculum for second year apprentices includes completing a skin anomaly course. Further, the use of a third year class as the baseline group instead of another second year class was due to the absence of other beauticians curriculum in the area. Additionally, two teachers were involved in the intervention, data collection and participated in an interview.

\subsection{Outline of the Study}

In January 2020, the experimental and baseline groups were exposed to the same pre-test on the recognition of elements present in skin anomaly images. From February 2020 to June 2020, only the experimental group attended the annotation and description training using the platform Realto via the videoconferencing software Zoom. In June 2020, the experimental group was exposed to a post-test (identical to the pre-test). Further, the experimental group was asked to answer a questionnaire on their perceptions of the usefulness of using annotations and descriptions to foster visual expertise, as well as their experience with the annotation tool of Realto. Finally, the experimental group was asked to take part in a discussion on their experience with visual expertise in the profession, the use of annotations and descriptions, and their general experience with the platform Realto. 


\subsection{Materials}

\subsubsection{Pre-Test and Post-Test}

All materials were cocreated with the teachers, including the pre-test, post-test and training activities. The pre-test and post-test are identical and focused on skin analysis, which is the main topic apprentices must learn about in their second year. In the test, the participants were first asked to observe and provide written descriptions of images depicting different body parts (Figure 3 - left). Then, they were asked to observe and provide written descriptions of images depicting sections of faces (e.g., cheeks, mouth) affected by skin anomalies (Figure 3 - centre). Finally, they were asked to observe and provide written descriptions of images of faces affected by conditions (e.g., young woman, middle-aged man, old woman) (Figure 3 - right). All the sections of the test prompted the apprentices to analyse the skin as professionally as possible, as if they were in front of a real client and performing a skin analysis (or anamnesis).
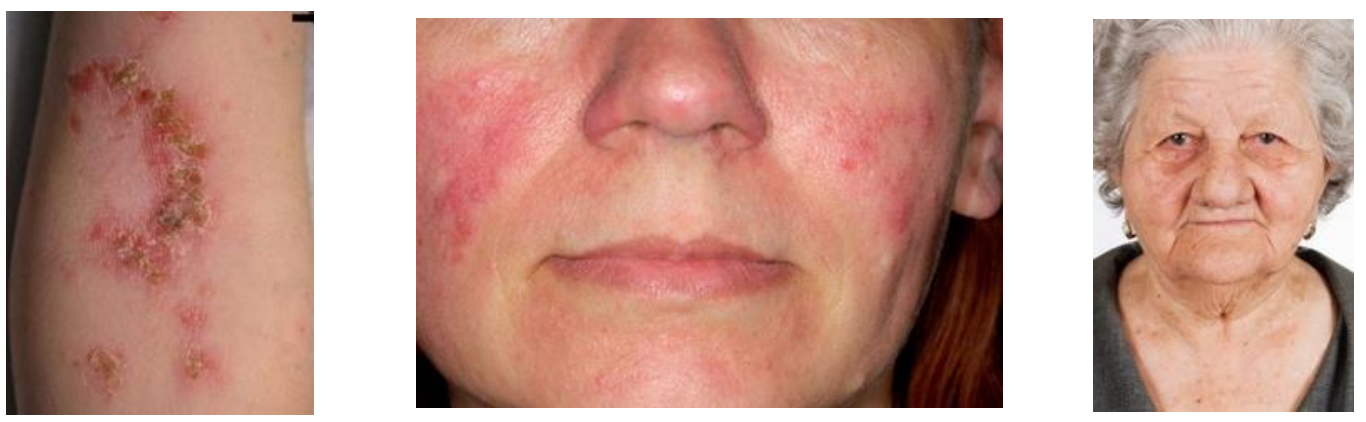

Figure 3. Arm affected by psoriasis (left), section of a face affected by rosacea (centre) and face of a woman with different sign of aging (right)

The scores of the pre-test and post-test were based on the written professional descriptions of the images provided by the teachers who codesigned the study. The teachers' descriptions of the 11 images contained 103 details (e.g., inflammation, spots, blisters, dilated pores) and covered all skin anomalies present in their school curriculum that beauticians must identify and treat (e.g., dry skin), refer to a doctor (e.g., psoriasis) or, in some cases (e.g., age spots), propose a correction for with make-up (e.g., foundation). Each apprentice's score was calculated by attributing one point to each detail found in their description that was also present in the teachers' descriptions and then summing them. In contrast, any missing or misrecognised detail was attributed zero points.

The pre-test and post-test were created while taking the experience of beauticians in the workplace into consideration as well as the final apprentice exam, when third year students have to perform a detailed skin analysis of a potential client.

\subsubsection{Questionnaires}

The questionnaire was composed of items from the Usefulness, Satisfaction \& Ease of Use (USE) Questionnaire (Lund, 2001) and other items designed specifically for the current study to measure the perceived usefulness of annotations and their impact on visual expertise as well as the perceived usefulness of descriptions. The participants had to indicate on a five-point Likert scale how much they agreed with the affirmative assertions that were successively proposed.

- The usefulness scale (Cronbach's $\alpha=$.936) of Lund's (2001) USE questionnaire served to assess the apprentices' perceptions of the usefulness of the annotations (e.g., "Annotations helped me be more effective").

- Two sets of 11 items were designed to measure the perceived impact of annotations on visual expertise. The first 11 items (Cronbach's $\alpha=.886$ ) referred to the annotations carried out by the teacher (e.g., "The teacher's annotation helped me identify textures specific to a given anomaly"), while the second 11 items (Cronbach's $\alpha$ $=.926$ ) referred to annotations carried out directly by the apprentices (e.g., "My annotations helped me identify textures specific to a given anomaly"). These two sets were inspired by analogous research in the area of visual expertise (see Chamberlain \& Wagemans, 2015; Ko et al., 2019; Lowenstein et al., 2019). These items were designed to facilitate understanding of whether the annotations affected the apprentices' visual expertise on a whole image and its details, the texture of anomalies and tissues, and some details they had not seen in that image before or previously had not considered relevant.

- One set of 3 items (Cronbach's $\alpha=.658$ ) was created to measure the apprentices' perceptions of the usefulness of the descriptions (e.g., to "Integrate the visual information with the theory"). 


\subsubsection{Discussion Guide for the Interviews}

The discussion guide focused on expanding and deepening the topics already faced in the questionnaire and investigating in detail the apprentices' and teachers' experiences with both the activities and platform. The three macro-categories identified for the interviews were: a) the relevance of visual expertise in the profession, b) the perceived usefulness of annotations and descriptions in helping observations and c) the sample experience with the platform and its potential additional uses for the future.

The interviews with the learners lasted 49 minutes on average $(\min =43 \mathrm{~min}$; $\max =55 \mathrm{~min}$ ), while the individual interviews with the teachers lasted 33.5 minutes on average $(\min =27 \mathrm{~min} ; \max =40 \mathrm{~min})$. The interviews were conducted and recorded via the Zoom videoconferencing software. A content analysis (Miles \& Huberman, 1994) using NVivo was applied to the whole corpus. This process involved three steps: first, the interview data were transcribed verbatim; second, the most salient themes were identified, according to the macro-categories; third, the themes were further coded to create micro-thematic categories.

\subsubsection{The Realto Platform and its Image Annotation Tool}

All activities were performed using the learning platform Realto, which was developed as part of the Dual-T project. The platform was created with the objective of connecting and supporting the experiences that apprentices have in different learning environments, such as school, branch courses and the workplace. Realto's look and feel is similar to a social network, but it contains multiple features created specifically for dual education. Besides features oriented to sharing educational and workplace content with teachers and classmates, the features that are used by teachers and students most often are the activities. Realto offers multiple types of activities, such as multiple-choice questions, textual replies with uploaded files, image annotations and many others. The image annotation and textual reply activities are the ones used in this research. Image annotation activities are performed using the annotation tool, an image manipulation function through which users can open an image and apply annotations to it. Users can access the tool by clicking on an image and then pressing the brush icon, which is visible on the central top of the screen. Realto will then display the picture on a black background alongside the annotation tool bar. The annotation tool bar (Figure 4) contains all the typical functions available with other annotation applications (e.g., SketchBook Pro). Users can perform different actions using the tool, such as 1) draw free lines, 2) add shapes (square, arrows and circles), 3) change the colours of shapes, lines and text, 4) select lines, shape or text, 5) add text and change its size and 6) edit the transparency and layers of elements (e.g., overlay).

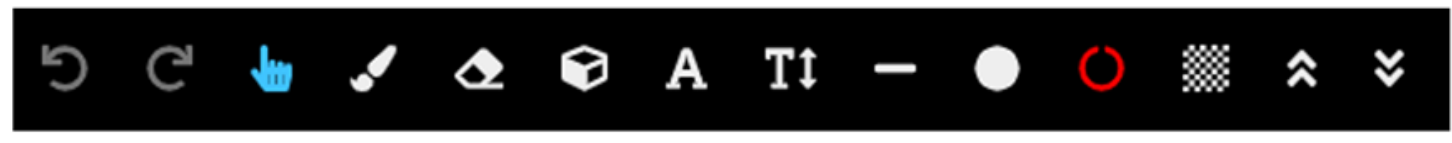

Figure 4. Realto's annotation bar with all its functions

\subsubsection{Procedure for the Activities}

Each lesson performed with the experimental group followed the procedure described below:

- Teacher-driven annotations: Each lesson began with the teacher uploading images of anomalies on Realto as a post and then sharing the screen with the students via Zoom. Then the teacher clicked on the images and proceeded to explain their relevant details while performing annotations (i.e., teacher-driven annotations), such as coloured marks or circles, to allow students to better understand the specific features of the disease, including differences in colours, variations in textures, and the presence of extraneous tissues or multiple anomalies (Figure 5). The teacher's annotations on the images were performed using the annotation function available for every image uploaded to the platform.
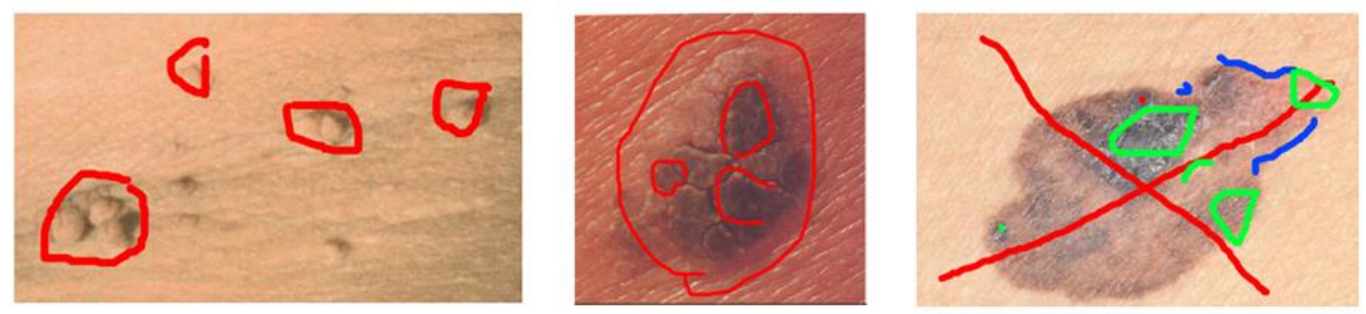

Figure 5. Teacher-driven annotations on different anomalies

- Students' annotations and written descriptions: The lesson continued with the teacher launching an activity, and 
asking the students to open the activity and carry it out in the appropriate section of Realto. To view the activities, apprentices had to go to the section of the platform where all the activities created by the teacher are stored. The exercises contained the images related to the topic explained by the teacher in the first part of the lesson. The students were first told to "Observe this anomaly as if it were the skin of the client. Use the annotation functions to signal the anomaly or the anomalies present in the image. Also, try to annotate all the main features present in the image, such as deformations, colours, shapes and textures or other abnormal aspects of the image." After that, the students were asked to "Write a complete description of the image referring to deformations, colours, shapes and textures or any other abnormal aspect of the image." By clicking on the correct activity, the students are able to view the description of the task and the images. Afterwards, apprentices could click on the images and annotate them, and click on the available text box to complete the descriptions.

- Correction of the activities: After all the students had completed the activities, the teacher opened the exercises in the results section of the activities and shared her screen. Realto allows teachers to display all the students' annotations overlapped on the same image and check or uncheck (i.e., visualise or not) a single participant's contribution. Apprentices had the chance to look at their own annotations that were performed on each of the images in the exercise as well as those of their classmates (Figure 6). Similarly, the teacher was able to open the textual replies and comment on the results of all the students. During the correction period, the students were invited to ask questions and reflect on the elements that were correctly identified, the ones that were missed, and the work of their classmates.

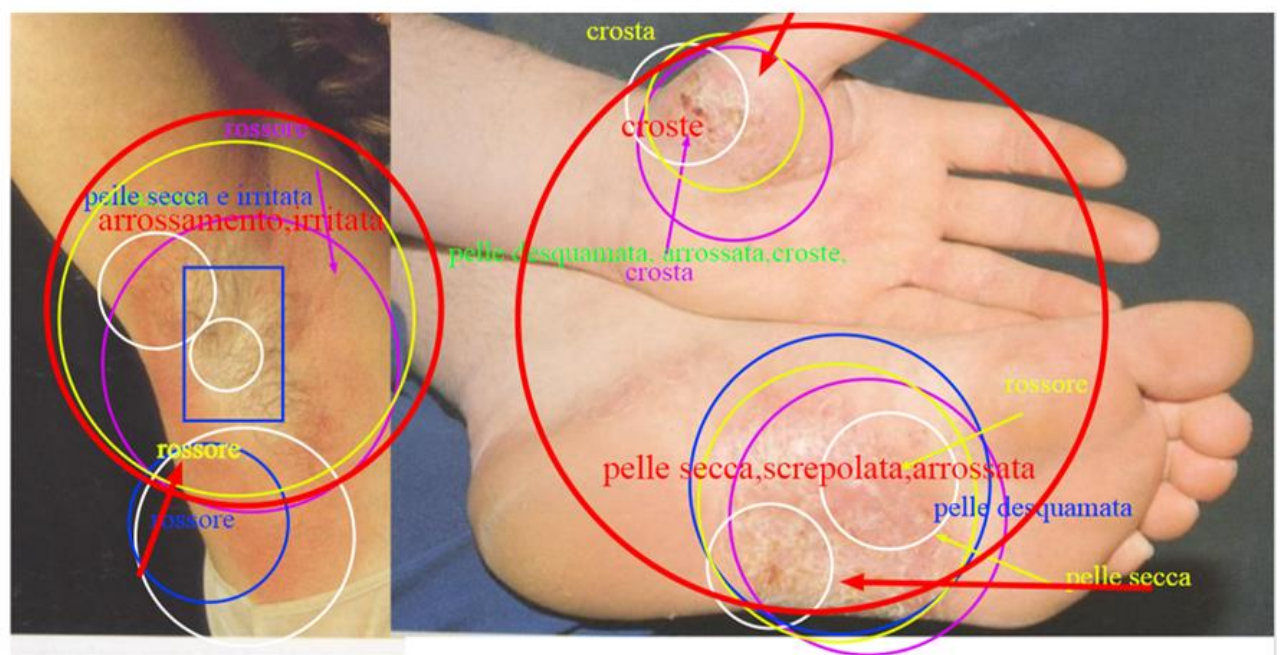

Figure 6. Students' overlapped annotations during correction

\section{Results}

\subsection{Pre-Tests and Post-Tests for the Written Descriptions}

A Wilcoxon signed-rank test was run on the pre-test and post-test written description scores of the experimental group. The post-test ranks $(\mathrm{Mdn}=28.00, \mathrm{SD}=3.23)$ were higher than the pre-test ranks $(\mathrm{Mdn}=14.00),(\mathrm{Z}=2.371, \mathrm{p}=.018$, $\eta 2=.80$ ). This confirms the direct impact of the activities on the number of details present in the descriptions. Further, a Mann-Whitney U test was run on the post-test (which was the only one carried out for the baseline group) scores of the experimental and baseline groups. The results indicated that at the post-test stage, the experimental group wrote significantly more details $(\mathrm{Mdn}=28.00, \mathrm{SD}=6.14)$ compared with the baseline group $(\mathrm{Mdn}=16.00)(\mathrm{U}=6.50, \mathrm{p}=.001$, $\eta 2=.47$ ), suggesting a positive impact as a result of the treatment (Figure 7). 


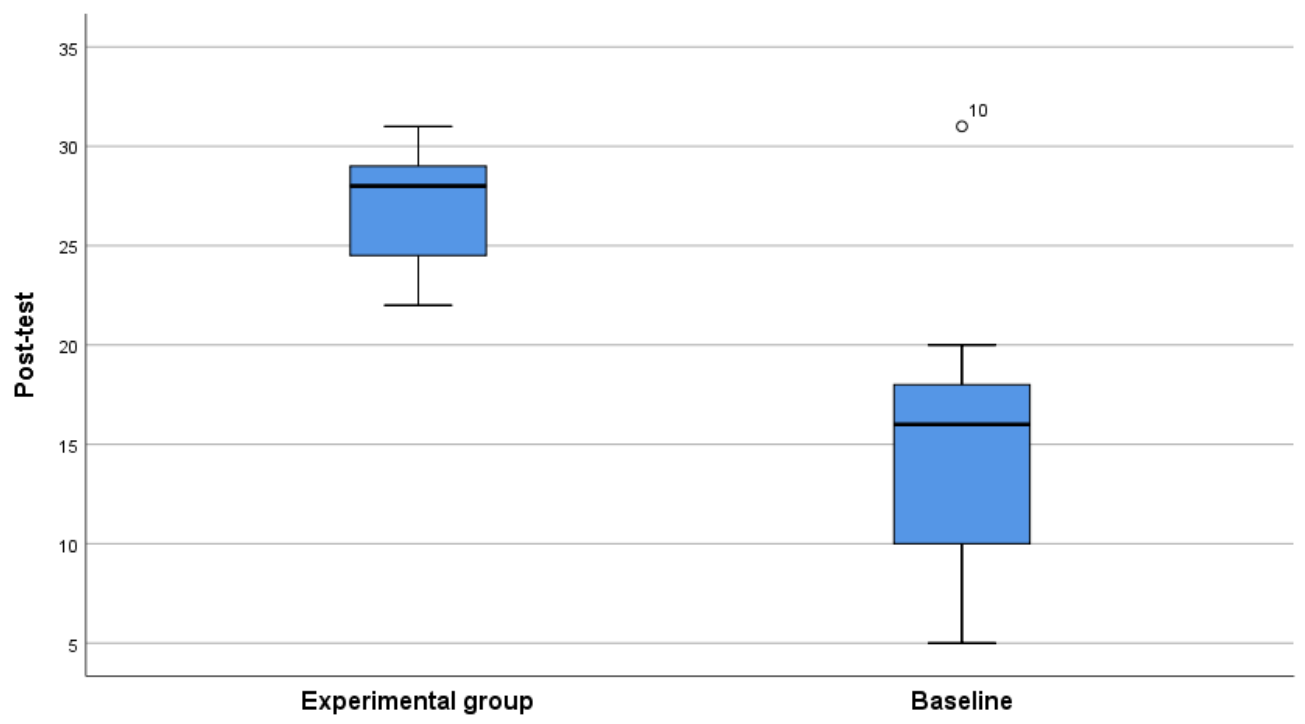

Figure 7. Scores of the experimental and baseline groups at the post-test stage

\subsection{Questionnaire}

\subsubsection{How Useful Do Apprentices Perceive Annotations to Be?}

The replies mostly ranged between "agree" and "strongly agree," while a few chose "neither disagree nor agree" or "disagree." The only item that strayed from the rest was "Time-saving," for which some of the participants expressed a slightly more negative outlook, with $44.50 \%$ choosing "agree," $22.20 \%$ selecting "neither disagree nor agree," and 33.30\% selecting "disagree" (Figure 8).

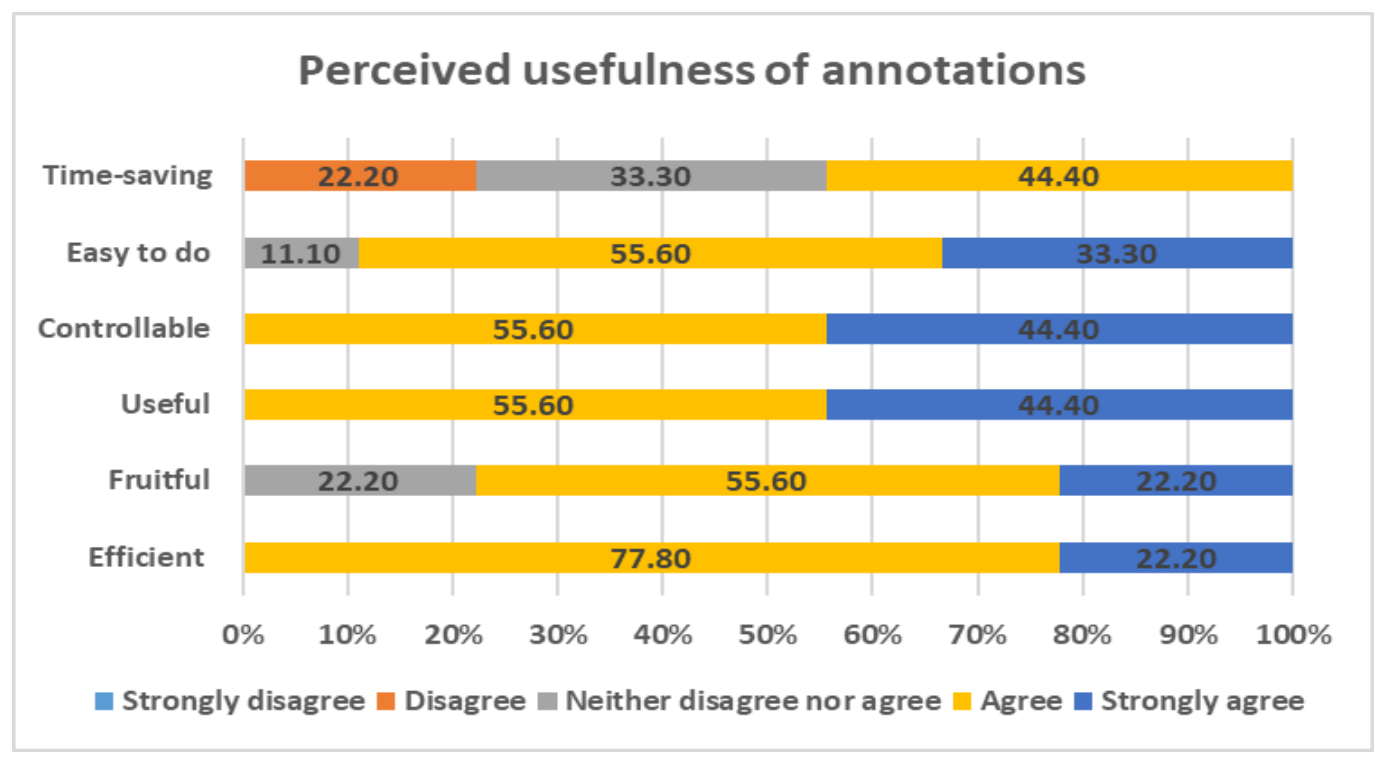

Figure 8. Perceived usefulness of annotations

\subsubsection{What is the Apprentices Perceived Impact of Annotations on Visual Expertise?}

A Wilcoxon signed-rank test was run to compare the scores given by the students to their own annotations, as well as the scores given to the teacher's annotations. The results indicate that the students' own annotations ranked significantly higher compared with those of the teachers $\left(Z=2.214, p=.027 ; \eta^{2}=.54\right)$. However, the participants gave both the students' annotations ( $\mathrm{Mdn}=4.46, \mathrm{SD}=.46)$ and teacher's annotations $(\mathrm{Mdn}=4.27, \mathrm{SD}=.37)$ high scores falling in the range of "agree." Therefore, students perceived their own annotations as affecting their own visual expertise more than the teacher's annotations, although they considered both types of annotations to positively affect their visual expertise (Figure 9). 


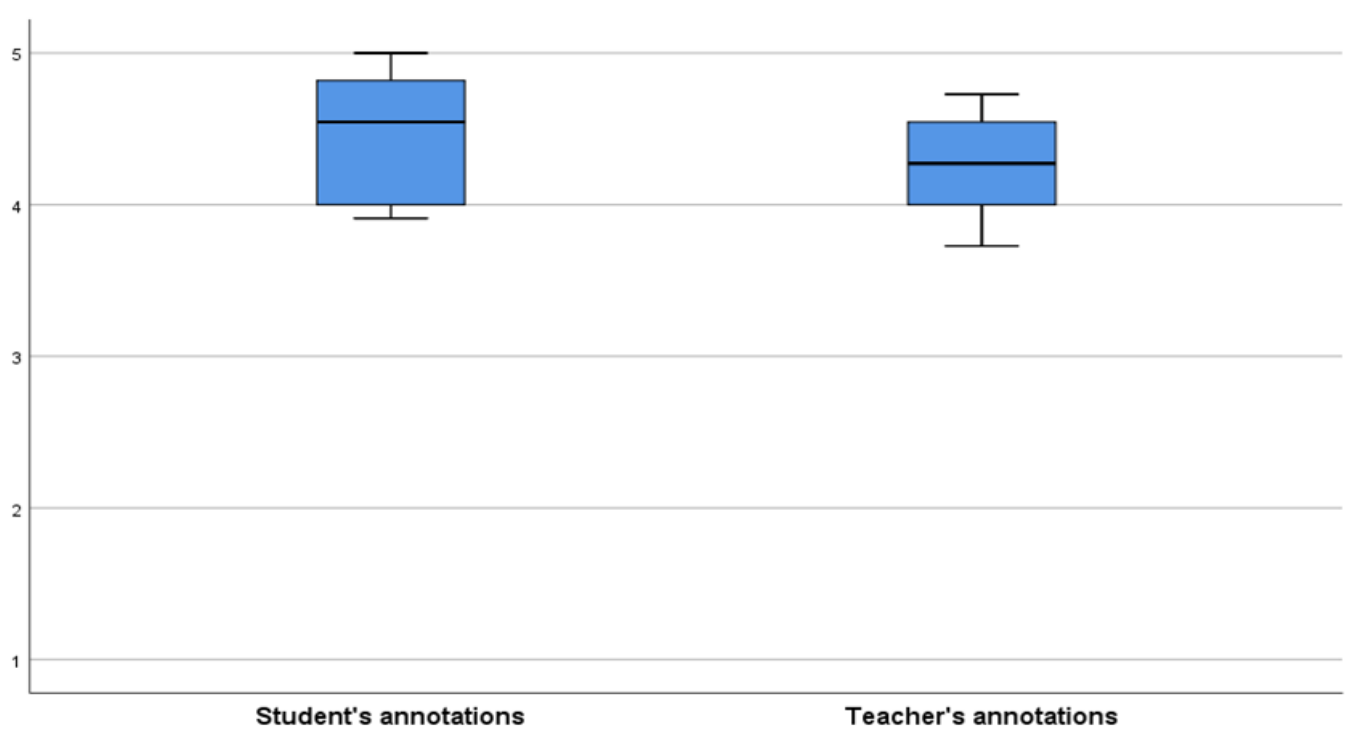

Figure 9. Apprentices' perceived impact of annotations on visual expertise

\subsubsection{How Useful do Apprentices Perceive Descriptions to Be?}

The replies to the question were positive and mostly ranged between "agree" and "strongly agree," with only $11 \%$ of replies indicating "neither disagree nor agree" for the item "Helped remembering things." This result implies the importance of descriptions for students because they helped them assimilate the content of the images (Figure 10).

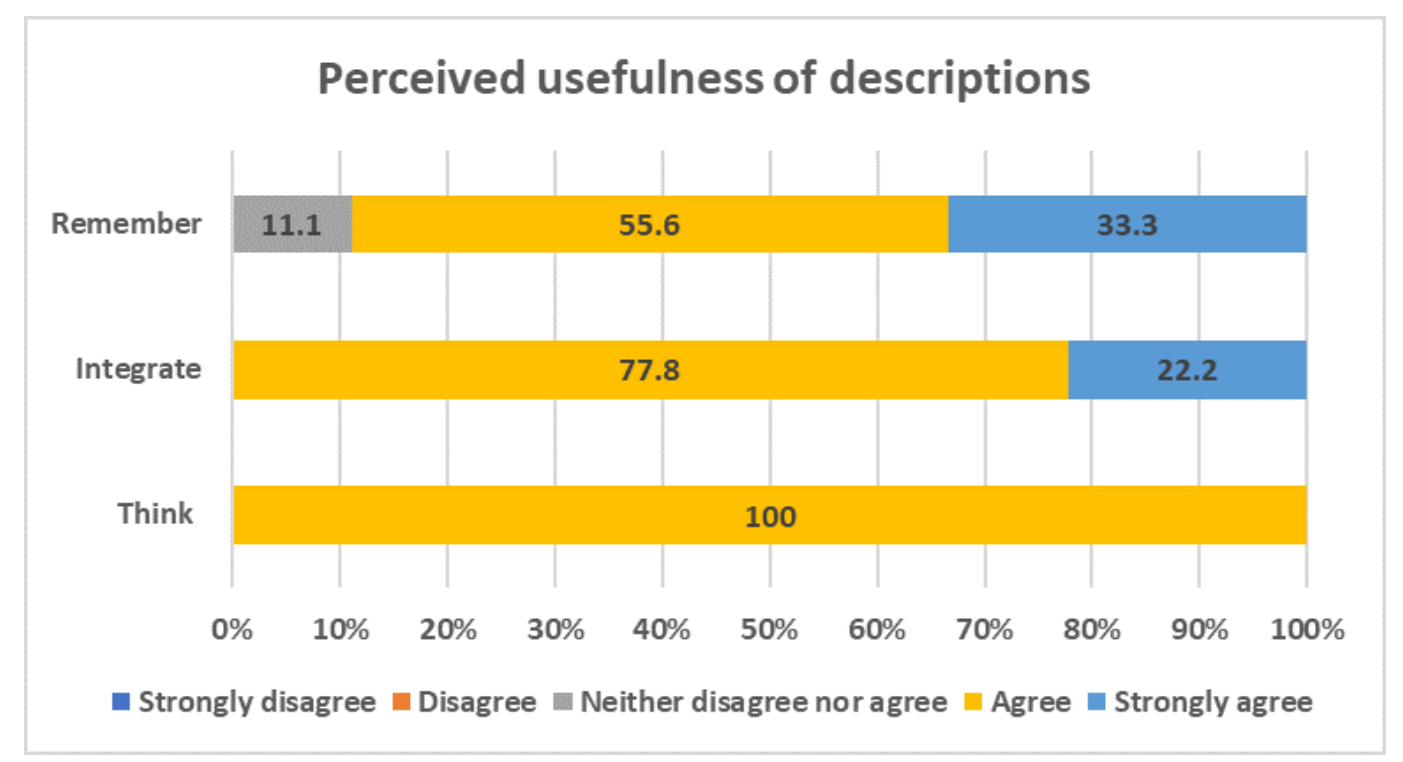

Figure 10. Perceived usefulness of descriptions

\subsubsection{How do Apprentices Perceive the USE of the Annotation Tool?}

The mean scores of the sample for the four factors of the USE Questionnaire (i.e., usefulness, satisfaction, ease of use and ease of learning) show that the participants gave scores that ranged from "strongly disagree" to "neither disagree nor agree". The factor with the highest score was "Usefulness," with a mean of 3.96, while "Satisfaction" scored the lowest, with a mean of 3.20 (Figure 11). However, in this case, no score was in a negative range. 


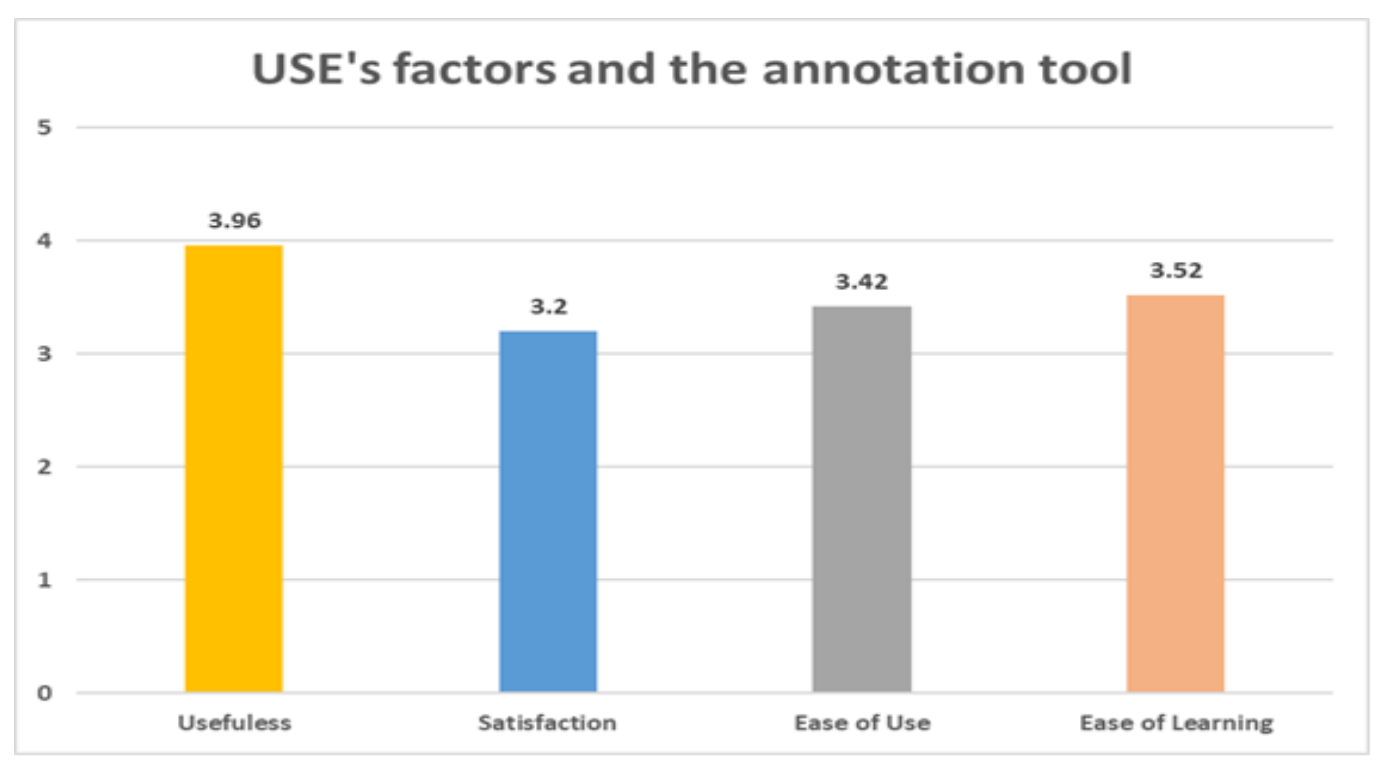

Figure 11. Mean scores for the four USE factors for the annotation tool

\subsection{Group (Apprentices) and Individual (Teacher) Interviews}

\subsubsection{Relevance of Visual Expertise in the Profession}

The apprentices reported knowing what they should do at work, but indicated that in most cases, they ended up writing down the anomalies and carried on with the treatment (1). However, the teachers explained how to observe, stressing the importance of performing skin analysis as follows (2):

(1) "We should check every inch of the skin and note down the anomalies. But in most cases, we just write down the main things on the folder of the client and we keep it in mind during treatment." (Apprentice_Chiara)

(2) "It's important not to mix up anomalies since they look similar. Before treating the skin, it's important to evaluate its status. We start observing from the top and then we identify the phototype of the skin, shape of the face, type of skin, and skin surface. If I see inflammation on the face, I can't, for instance, colour the eyebrows if the skin is red!" (Teacher_Martina)

\subsubsection{Usefulness of the Annotations}

The apprentices indicated that performing and seeing annotations during correction is useful. Also, they underlined that a teacher's annotations coupled with an explanation of the anomaly are essential to understand what anomalies look like (3). Further, the teachers reported a positive experience with the annotations as follows, and considered them to have helped apprentices observe, explore and be more attentive (4):

(3) "It is useful. I like the teacher's annotations since she explains the image, like how moles look like and how dangerous they are. Also, she first explains it and then points towards the areas we must look at and evaluate. Without her explanation, I'd not know where to start. I also like seeing my classmates' annotations!" (Apprentice_Francesca)

(4) “Annotations helped with identifying certain elements by 'keeping their eyes peeled.' Annotations impacted visual expertise and students appreciated it and, at the end of the course, they managed to do a proper evaluation of the skin. They can identify the anomalies now since they know which anomalies share similar features." (Teacher_Valentina)

\subsubsection{Usefulness of the Descriptions}

The descriptions were considered complex to perform (5), but made the apprentices attentive (6) and helped with visual expertise as follows (7):

(5) "Describing images can be complex since I really have to look at the image to be able to describe it properly." (Apprentice_Francesca)

(6) "I've become more focused when I describe images" (Apprentice_Chiara)

(7) "Descriptions were extremely helpful for visual expertise, in addition to annotations. Before we did similar activities on paper, but with Realto, we kept repeating this activity with new anomalies, and it really helped 
apprentices' visual expertise and identification." (Teacher_Valentina)

\subsubsection{Use of Realto in the Future}

The apprentices were positive about Realto, but suggested changes in the type of activities to carry out with the platform and more challenging tasks (8). The teachers were also positive and already planned to modify some activities for the next year, as described below (9).

(8) "We should continue with image annotations and descriptions of anomalies of the entire face, but maybe fewer annotations of images with just one big anomaly. It can be too obvious with that." (Apprentice_Chiara)

(9) "Future activities with Realto could continue with the use of descriptions and then also use of visual annotations, but also text annotations. In this case, they don't stop at marking one area, but they already have to name the section of the image and have to connect things." (Teacher_Martina)

\section{Discussion and Conclusions}

The aim of the current study was to explore the use of annotations to foster visual expertise in apprentices beauticians. The main hypothesis was that the apprentices exposed to teacher-driven annotations, student-driven annotations and description activities would be able to recognise more details in an image analysis task compared with those students trained in the traditional way. To achieve this aim, a group of apprentices beauticians $(\mathrm{N}=9)$ of the second year were firstly exposed to a pre-test on skin anomaly analysis, then to the training sessions for a semester and concluded with a post-test, a questionnaire and the participation to a group interview. Conversely, a class of the third year $(\mathrm{N}=19)$, who already completed the traditional course, was used as a baseline group.

The results of the written descriptions from the pre-test and post-test stages showed a significant difference between the groups, with the experimental almost doubling the amount of details written by the baseline group. This indicates that teacher-driven annotations combined with the activities: 1) positively influenced the apprentices and 2) led them to observe the relevant parts of the image, helping them to recognise and name skin anomalies. Further, the results of the experimental group indicate that a combination of teacher-driven annotations, student-driven annotations and written descriptions of images depicting anomalies were more powerful in training students about skin anomalies when compared with a traditional course. This can be seen by comparing their scores to those of the baseline group; although the baseline group was trained in the regular way during their second year and continued exercising visual expertise during the first part of the third year of their training, they wrote fewer details than the experimental group. It is also important to note that the teacher's descriptions contained a total of 103 written details, a score that is much higher than the students' scores. This confirms the results of other trainings on multiple exposures to anomaly images (e.g., Dolev et al., 2011) and diagnostic accuracy. Future studies in this area could make use of other kinds of signals (e.g., Jarodzka et al., 2013) and include more objective methodologies, such as eye-tracking.

The results of the questionnaires are also positive overall. In the first question about the perceived usefulness of annotations, most of the participants considered using annotations as being useful, efficient, controllable, easy to do and fruitful, even if they pointed out that it might not save them a lot of time. Similarly, answers to the items related to the perceived impact of annotations on visual expertise indicated that annotations helped apprentices observe images in their totality and in detail. Almost all participants agreed that annotations helped them identify specific anomalies, tissues, discolorations, textures and details that were previously not considered relevant or seen in specific images. Plus, the results of the Wilcoxon signed-rank test indicated that the students perceived their own annotations as more helpful for observing images than the teacher's. In general, the apprentices were helped by both their own annotations and those of the teacher while observing the images. Because this section of the questionnaire was modelled on the work of Ko at al. (2019) and Lowenstein et al. (2019), it is important to point out that the students' positive replies indicate that annotations not only allowed them to observe images in a more general way, but also more specifically. Annotations fostered the identification of features such as colours, textures and specific tissues in the anomaly, and were useful in identifying elements not previously seen or considered relevant. At the same time, despite the positive results of the questionnaire, it is interesting to read these results in light of the group interviews, in which students put a greater emphasis on the impact of the teacher's annotations and use of descriptions. It is possible that the students might have undervalued the impact of their own annotations when it comes to visual expertise. Similar results were also seen from the items on the perceived usefulness of descriptions, with most of the students agreeing that the descriptions allowed them to think about the images, integrate concepts, and memorise them. Interestingly, this result could provide an indication as to where to focus this kind of training. For instance, in the activities, a section on metacognition could be implemented that focuses on solidifying integration, memorisation and recall for different anomalies and their features.

In the group interview, the apprentices reported that they did not follow a specific way of observing the client's skin. Conversely, the teachers strongly felt the necessity to check the skin to find anomalies, warn the client in case of dangerous 
anomalies and check the status of treatment, indicating a more structured way of carrying out professional visual expertise. As shown in the questionnaire, the apprentices generally had a positive perception of annotations and descriptions in visual expertise tasks, but they indicated that annotations are more beneficial in exercises, with the images depicting a full face compared with just an affected body part. The teachers recognised the didactical relevance of using annotations to highlight certain elements of the image and enhance the attention of the students while increasing their ability to recognise anomalies. The students also mentioned the importance of annotation to practice because they did not always have this chance at work.

The descriptions were useful for increasing attention and forcing the students to observe more intensively since they had to describe the images. Further, the apprentices indicated that the descriptions made them more focused, even if they were challenging. The teachers were positive about the descriptions and noted that they helped in supporting the visual expertise, identification and internalisation of the content. This also suggests that combining annotations and descriptions constitutes a powerful option for teaching about anomalies.

Both groups showed interest in continuing to use Realto, but with a slightly different format. For instance, the students pointed out that they preferred to use Realto during all the lessons, but for a shorter time. The teachers explained that they would like to explore the use of written annotations more.

Although the results are positive, it is important, to make some limitations of the study explicit. The first is surely due to use of the third year class (who already complete skin anomaly course) as a baseline group. Having two classes of the same year would have been a better choice but no other second year class was available in the area for the intervention. Another issue is related to the sample size of the experimental group which was rather small; having a larger sample could allow for more fine-grained analysis and more complex experimental designs. The small sample size limits the generalizability of the data but reflects the actual number of apprentices enrolled in the certificate and in that specific year.

Besides this limitation, we believe the results could be relevant for the general research on visual expertise and for the development of the profession and practitioners. Our contribution to the field can be incorporated in three main points:

- The positioning of the current research in vocational and education and training (VET) instead of higher education.

- The need of the apprentice beauticians to enhance their currently limited-in-time training on the visual expertise and identification of skin anomalies to become more competent professionals.

- The training material created for the present study combines elements of other trainings that already proved to be effective in other fields, such as1) the use of attention-guiding cues to train students' gaze (e.g., Boucheix \& Lowe, 2010), 2) the use of technology-supported annotations to help professional dermatologists with skin anomalies (e.g., Ferreira et al., 2012; Eapen et al., 2020) and 3) repeated sets of images (e.g., Rimoin et al., 2015).

This research could open new ways to train visual expertise skills in many vocational professions. For instance, massage therapists could be trained to observe the patient's body in a more specific way and identify anomalies (e.g., rotation of the pelvis, pronation, etc.) that the patient is not yet aware of and that newcomers to the field might take time to recognise. Also, professions such as, for example, gardeners could improve their visual expertise of similar plants and anomalies (plant protection) that attack plants and that share similar features. In addition, apprentices in technical professions could benefit from training in technical drawings. There is a wide range of possibilities that are worth exploring with methods that include annotations and the description of images.

\section{Funding}

This work is supported by the Swiss State Secretariat of Education, Research and Innovation (SERI) [1315000851].

\section{Acknowledgements}

We would like to thank Prof. Jean-Luc Gurtner, Ms. Claudia Berri and Ms. Luisa Broggini.

\section{References}

Caruso, V., Cattaneo, A., Gurtner, J. L., \& Ainsworth, S. (2019). Professional vision in fashion design: practices and views of teachers and learners. Vocations and Learning, 12(1), 47-65.

Boucheix, J. M., \& Lowe, R. K. (2010). An eye tracking comparison of external pointing annotations and internal continuous annotations in learning with complex animations. Learning and Instruction, 20(2), 123-135. https://doi.org/10.1016/j.learninstruc.2009.02.015

Brown, N. H., Robertson, K. M., Bisset, Y. C., \& Rees, J. L. (2009). Using a structured image database, how well can novices assign skin lesion images to the correct diagnostic grouping? The Journal of Investigative Dermatology, 129(10), 2509-2512. https://doi.org/10.1038/jid.2009.75 
Chamberlain, R., \& Wagemans, J. (2015). Visual arts training is linked to flexible attention to local and global levels of visual stimuli. Acta Psychologica, 161, 185-197. https://doi.org/10.1016/j.actpsy.2015.08.012

D'Innocenzo, G., Gonzalez, C. C., Williams, A. M., \& Bishop, D. T. (2016). Looking to learn: The effects of visual guidance on observational learning of the golf swing.Plos One, 11(5), e0155442. https://doi.org/10.1371/journal.pone.0155442

De Koning, B. B., Tabbers, H. K., Rikers, R. M., \& Paas, F. (2007). Attention cueing as a means to enhance learning from an animation. Applied Cognitive Psychology: The Official Journal of the Society for Applied Research in Memory and Cognition, 21(6), 731-746. https://doi.org/10.1002/acp.1346

State Secretariat for Education, Research and Innovation. (2007). Ordinanza della SEFRI sulla formazione professionale di base Estetista con attestato federale di capacità (AFC). Retrieved from: https://www.fedlex.admin.ch/eli/cc/2007/68/it

Dolev, J. C., O'Sullivan, P., \& Berger, T. (2011). The eDerm online curriculum: A randomized study of effective skin cancer teaching to medical students. Journal of the American Academy of Dermatology, 65(6), e165-e171. https://doi.org/10.1016/j.jaad.2010.07.024

Eapen, B. R., Archer, N., \& Sartipi, K. (2020). LesionMap: A method and tool for the semantic annotation of dermatological lesions for documentation and machine learning. JMIR Dermatology, 3(1), e18149. https://doi.org/10.2196/18149

Ferreira, P. M., Mendonça, T., Rozeira, J., \& Rocha, P. (2012). An annotation tool for dermoscopic image segmentation. In Proceedings of the 1st International Workshop on Visual Interfaces for Ground Truth Collection in Computer Vision Applications, 1-6. https://doi.org/10.1145/2304496.2304501

Friedman, R. J., Rigel, D. S., \& Kopf, A. W. (1985). Early detection of malignant melanoma: The role of physician examination and self-examination of the skin. CA: A Cancer Journal for Clinicians, 35(3), 130-151. https://doi.org/10.3322/canjclin.35.3.130

Heiser, J., \& Tversky, B. (2006). Arrows in comprehending and producing mechanical diagrams. Cognitive Science, 30(3), 581-592. https://doi.org/10.1207/s15516709 $\operatorname{cog} 0000 \_70$

Jarodzka, H., Van Gog, T., Dorr, M., Scheiter, K., \& Gerjets, P. (2013). Learning to see: Guiding students' attention via a model's eye movements fosters learning. Learning and Instruction, 25, 62-70. https://doi.org/10.1016/j.learninstruc.2012.11.004

Katsioloudis, P. J., \& Stefaniak, J. E. (2018). Effectiveness of drafting models for engineering technology students and impacts on spatial visualization ability: An analysis and consideration of critical variables. Journal of Technology Education, 29(2). http://doi.org/10.21061/jte.v29i2.a.6

Kellman, P. J., \& Krasne, S. (2018). Accelerating expertise: Perceptual and adaptive learning technology in medical learning. Medical Teacher, 40(8), 797-802. http://doi.org/10.1080/0142159X.2018.1484897

Ko, C. J., Braverman, I., Sidlow, R., \& Lowenstein, E. J. (2019). Visual perception, cognition, and error in dermatologic diagnosis: Key cognitive principles. Journal of the American Academy of Dermatology, 81(6), 1227-1234. http://doi.org/10.1016/j.jaad.2018.10.082

Kredel, R., Vater, C., Klostermann, A., \& Hossner, E. J. (2017). Eye tracking technology and the dynamics of natural gaze behavior in sports: A systematic review of 40 years of research. Frontiers in psychology, 8, 1845. https://doi.org/10.3389/fpsyg.2017.01845

Kyriakou, G., \& Kyriakou, A. (2021). More than meets the eye (brow): Dermatologic imagery in Frida Kahlo's selfportraiture. Clinics in Dermatology, 39(1), 146-148. http://doi.org/10.1016/j.clindermatol.2020.07.004

Lowenstein, E. J., Sidlow, R., \& Ko, C. J. (2019). Visual perception, cognition, and error in dermatologic diagnosis: Diagnosis and error. Journal of the American Academy of Dermatology, 81(6), 1237-1245. http://doi.org/10.1016/j.jaad.2018.12.072

Lund, A. M. (2001). Measuring usability with the USE questionnaire. Society for Technical Communication. http://www.stcsig.org/usability/newsletter/0110_measuring_with_use.html

Mayer, R. E. (2002). Multimedia learning. Psychology of Learning and Motivation, 41, 85-139. https://doi.org/10.1016/S0079-7421(02)80005-6

McGrath, C., McCourt, C., Corry, A., Dounavi, K., Dillenburger, K., \& Gormley, G. (2018). The use of 'precision teaching' in enhancing medical students' dermatological diagnostic skills. MedEdPublish, 7. 
https://doi.org/10.15694/mep.2018.0000091.1

Miles, M. B., \& Huberman, A. M. (1994). Qualitative data analysis: An expanded source book (2nd ed.). Sage.

Naghshineh, S., Hafler, J. P., Miller, A. R., Blanco, M. A., Lipsitz, S. R., Dubroff, R. P., Khoshbin, S., \& Katz, J. T. (2008). Formal art observation training improves medical students' visual diagnostic skills. Journal of General Internal Medicine, 23(7), 991-997. https://doi.org/10.1007/s11606-008-0667-0

Ravesloot, C., van der Schaaf, M., Haaring, C., Kruitwagen, C., Beek, E., Ten Cate, O., \& van Schaik, J. (2012). Construct validation of progress testing to measure knowledge and visual skills in radiology. Medical Teacher, 34(12), 10471055. https://doi.org/10.3109/0142159X.2012.716177

Rimoin, L., Altieri, L., Craft, N., Krasne, S., \& Kellman, P. J. (2015). Training pattern recognition of skin lesion morphology, configuration, and distribution. Journal of the American Academy of Dermatology, 72(3), 489-495. https://doi.org/10.1016/j.jaad.2014.11.016

Scheiter, K., Eder, T., Richter, J., Hüttig, F., \& Keutel, C. (2019). Dental medical students' competencies for identifying anomalies in X-rays: When do they develop? (Conference paper). 18th Biennial Conference of the European Association for Research on Learning and Instruction (EARLI), Aachen, Germany.

Schneider, S., Beege, M., Nebel, S., \& Rey, G. D. (2018). A meta-analysis of how signalling affects learning with media. Educational Research Review, 23, 1-24. https://doi.org/10.1016/j.edurev.2017.11.001

Shapiro, J., Rucker, L., \& Beck, J. (2006). Training the clinical eye and mind: Using the arts to develop medical students' observational and pattern recognition skills. Medical Education, 40(3), 263-268. https://doi.org/10.1111/j.13652929.2006.02389.x

Shin, D., \& Park, S. (2019). 3D learning spaces and activities fostering users' learning, acceptance, and creativity. Journal of Computing in Higher Education, 31(1), 210-228. https://doi.org/10.1007/s12528-019-09205-2

Stefanut, T., \& Gorgan, D. (2008). Graphical annotation based interactive techniques in eTrace eLearning environment. In Proceedings of the 4th International Scientific Conference eLSE, 17-18. https://doi.org/10.1007/978-3-54089350-9_11

Styhre, A., \& Gluch, P. (2009). Visual representations and knowledge-intensive work: The case of architect work. The Journal of Information and Knowledge Management Systems, 39(2), 108-124. https://doi.org/10.1108/03055720910988832

van Gog, T. (2014). The signaling (or cueing) principle in multimedia learning. In R. E. Mayer (Ed.), The Cambridge Handbook of Multimedia Learning (pp. 263-278). Cambridge University Press. https://doi.org/10.1017/CBO9781139547369.014

Waite, S., Grigorian, A., Alexander, R. G., Macknik, S. L., Carrasco, M., Heeger, D. J., \& Martinez-Conde, S. (2019). Analysis of perceptual expertise in radiology - Current knowledge and a new perspective. Frontiers in Human Neuroscience, 13, 213. https://doi.org/10.3389/fnhum.2019.00272

Zanotto, M., Ballerini, L., Aldridge, B., Fisher, R. B., \& Rees, J. (2011). Visual cues do not improve skin lesion ABC(D) grading. Proc. SPIE 7966, Medical Imaging 2011: Image Perception, Observer Performance, and Technology Assessment, 79660U. https://doi.org/10.1117/12.878115

Zimmermann, C., Huang, J. T., \& Buzney, E. A. (2016). Refining the eye: Dermatology and visual literacy. Journal of Museum Education, 41(2), 116-122. https://doi.org/10.1080/10598650.2016.1163189

Zywica, J., \& Gomez, K. (2008). Annotating to support learning in the content areas: Teaching and learning science. Journal of Adolescent \& Adult Literacy, 52(2), 155-164. https://doi.org/10.1598/JAAL.52.2

\section{Copyrights}

Copyright for this article is retained by the author(s), with first publication rights granted to the journal.

This is an open-access article distributed under the terms and conditions of the Creative Commons Attribution license which permits unrestricted use, distribution, and reproduction in any medium, provided the original work is properly cited. 